\title{
Design of Electric Power Dispatching Management System based on Data Mining
}

\author{
Yanli Chang ${ }^{\mathrm{a}}$, Yanping Chen ${ }^{\mathrm{b}}$, Liruo Lu ${ }^{\mathrm{c}}$, Mengqing Jia ${ }^{\mathrm{d}}$ \\ State Grid Henan Electric Power Company Luoyang Power Supply Company, \\ Luoyang 471000, China \\ 43316856@qq.com, 1015903240@qq.com
}

Keywords: Electric power dispatching, Data Mining, Quality management.

\begin{abstract}
Electric Power is the vital energy source in national product and people daily life so the importance of electric power production and transmission is beyond question. As the link between electric power production and transmission, electric power dispatching decides the effective and healthy operation of the whole electric net. The automation and intelligent of electric power dispatching is a longtime task deserving thorough research. However, at present the electric power automation system served mostly for electric power production foreground while the background dispatching and production management work lack of complete technology support. Those problems how to harmonize the foreground control and background management in electric power production process, to construct a organic and uniform comprehensive intelligent system, to form perfect and smooth data stream and finally realize the integration and standardization of production plan, production process and improve the electric net management quality became burning problems.
\end{abstract}

\section{Introduction}

From the 1970s to the present, has gone from power dispatch to the grid smart grid monitoring safety warning long course of development, not only for power plants, substations and power transmission equipment for real-time monitoring, but also to monitor information from electrical equipment for analysis prediction, auxiliary power production decisions, to ensure stable power system operation, health, safety and reliable. In recent years, electricity production capacity and electricity load is increased compared with a decade ago, several times, and gradually formed a hybrid AC-DC power grid, the size and complexity of the scheduling management of the unprecedented increase in ultra-high pressure. Traditional and backward scheduling management model has been increasingly unable to meet the requirements of the rapid development of the power grid, it has seriously hampered the effective implementation of electricity production. The early 2000s, the State Grid Corporation requires each dispatch center construction Dispatch Management Information System (DMIS), whose functions cover to protect the value, maintenance scheduling, log management. As a landmark, it began the process of automation and information technology. Now, with the gradual deepening of the power system information construction, power grid with the urgent need to establish a national network regulation reconcile Unicom, provincial, prefecture-level and county-level business intelligence through efficient scheduling management system, grid integration of various departments and data streamline the production process, standardized to ensure the health power production and grid operation, sustained and stable development.

In management science, computer science and communication systems at the core of the scientific information system began in the 1950s, after half a century of development, has penetrated into all aspects of social production and civic life. As a leader in this field, University of Minnesota opened its information systems related courses in 1969, and set up a related research center. During 1981-1997, research information and management systems focused on the technical aspects of information systems, implementation and decision support, and its application is to study the existing manual operation of automation projects. Thereafter, the information system is 
gradually applied to the management of work, and with the development of electronic technology and network communication technology which gradually penetrated into electronic information systems and electronic data interchanges system.

Data mining technology successfully applied in insurance, marketing and insurance fields to make a good example for other areas of the power industry and then also introduces data mining technology. In contrast to other areas of data mining technology in the power industry is mainly limited to some specific data analysis and business decision aids on. Chinese scholars in the application of data mining technology for power system studies carried out, including: the analysis of power load based on data mining; analysis based on data warehousing and business data integration trend of electricity; power equipment defect analysis and processing.

\section{The Key Technologies of Power Dispatching Based on Data Mining}

Due to the special nature of the industry, power dispatching data warehouse has its distinctive features. Power dispatching more types of information systems, common ones include SCADA, EMS, relay protection and fault information system, the electricity market support systems, maintenance management systems, performance appraisal system and dispatch planning system, often there are frequent among these systems work, the need for complex data sharing and data exchange. Traditional relational database suitable for transaction processing, such as electrical equipment defects query, energy metering and management, and it is difficult to meet the electricity power supply for data analysis and decision support capabilities in these areas must be by means of a data warehouse and data mining technology.

Power dispatching data warehouse construction, on the one hand a clear business logic, data relationships clear, but on the other hand the lack of reference, it is appropriate to adopt a bottom-up approach to the design and implementation. First, a comprehensive analysis by means of electric power dispatch system identifies the data source, and then extracts the data source, forming a comprehensive power scheduling data warehouse, and gradually expand, refine and improve. Power dispatch warehouse building process you must first choose the theme, and then set the theme of the source data processing and integration. Power Data Warehouse data sources may include electricity load information, time information, weather information and other conventional data, these data sources through processing and integration into a data warehouse. In operation, data warehouse information must be kept up to date, depending on the monitor and the hub. Monitor for sensing and capturing update data source changes, when the source data changes, the monitor extraction of data according to the rules of data warehouse construction, and submit data to the Integrator. Integrator's responsibility is to monitor the captured data integrated conversion, input to update the data warehouse. Data warehouse through integrated data processing through data mining (DM) programs or other online analysis program (On-Line Analytical Processing, OLAP) analysis and extraction, to form more valuable high-level information for top-level client application analysis, decision to use.

Data Mining (Data Mining, DM) is an advanced data processing, and its goal is to extract valuable information to people implied data from the mass, and a people can understand the pattern shown. Knowledge of data mining data source technology processing may be incomplete, noisy and random, data mining techniques can be learned from these data sources of knowledge, and you can find new knowledge, and then to get to a form that people can understand representation out and storage and use. Because of the power of data mining, one appears to have been widespread attention, this technique is also known as KDD (Knowledge Discovery in Database, KDD), data mining technology show a close relationship with the database.

\section{The Functions of The Power Dispatch Management System}

Currently, electric power dispatch showing independent and redundant construction status, urban country have deployed their own scheduling management system, scheduling policies vary, the data also failed to achieve shared, direct impact on the efficiency of power dispatch, also impact on the development of electricity production plan. Therefore, it is necessary to design and implement a set 
of convenient, efficient and set provincial, city, county power dispatch management system integrated to form a complete, unified power dispatch system for the province's electricity and electricity production scheduling provided efficient decision support, improve the quality of power supply for the majority of users to provide better electricity supply services. Electric power dispatch system from the main function of the data service module, scheduling management module, production analysis module and video surveillance modules.

\subsection{Power Dispatching Data Platform}

Power dispatching data platform to collect data from the production site and scheduling system at all levels, and a standardized and unified data warehouse, centralized each subsystem provides business information and data services, as well as between different security zones located service module the data exchange channels and data bus. From the data source, the data collected on the transverse and longitudinal data. Transverse data refers to data from the same level of related business systems, such as: production management systems, power marketing system, power grid monitoring system, energy management system, wind power forecasting system, line monitoring system-level integrated planning systems. Longitudinal data refers to data from the upper and lower levels of the system, including: the power plant from the production management data, the data from all over the tone of metering systems and energy management systems.

\subsection{Dispatching Management}

Schedule management subsystem is the core of the power dispatch system, on the basis of power dispatching data platform, the device data from the Data Service data platform, the device management module is divided accordingly interval equipment, equipment classification and equipment storage, and construction equipment basic data; the use of equipment based on the data available for device data analysis, and provides statistical data users, data display and data query. Device management activities, construction equipment and the underlying database is the core focus. Building their own equipment from the underlying database and maintained at all levels, according to their respective jurisdictions, the front-line dispatch personnel for maintenance and device information entry, data entry via the back after the responsible leadership for approval of storage.

\subsection{Production Analysis}

Production is scheduled to run power analysis subsystem contains the analysis, secondary equipment analysis and safety analysis dispatch three functional modules, provides an evaluation of the quality of electricity production management scheduling, providing electricity scheduled to run in all directions, the entire process and supervision of all personnel . Comply with the requirements of the State Grid Corporation of electricity to run the risk controllable, controllability and the control target. Power dispatching analysis of data from the power dispatching data platform, it is operational control class data, scheduling class data and statistical analysis class data. Analysis period can be days, months or years. Dispatching analysis module based on different data sources, using different cycles, an analysis report of different frequencies.

\subsection{Video Surveillance}

Video surveillance systems for the safe operation of unmanned substations to provide a strong guarantee, a video surveillance system in a hierarchical structure-building, including three layers: the provincial control center, ground control center and station end system. Provincial control center by the access servers, disk arrays, Web servers, streaming media servers and videoconferencing gateway, whose function is to call control, multimedia information management, can achieve multimedia signal processing and forwarding, video conferencing with emergency command system interconnect and system permissions and parameters management.

\section{Summary}

With the development of the grid size, the number of stations to increasing the amount of data increases rapidly in power systems, data analysis and management of the increasing difficulty; at the same time, in addition to the electricity dispatching department, other departments and regulatory agencies also has data on the grid interest. So how massive power grid data analysis and 
processing, it ensures the safety and efficient implementation of the electricity production, but also able to provide decision support for power production and power scheduling, which is an important direction of the power of current research information systems.

\section{Reference}

[1]Bing Q I, Tang L R, Gong G J. Design of Electric Power Load Management System Based on Mobitex[J]. Automation of Electric Power Systems, 2006, 30(5):83-86.

[2]Cheng P G. Model Design of Information Management System Based on Data Mining[J]. International Conference on Signal Processing Systems, 2009:834-836.

[3]Liu J, Duan Y B. The design and implementation of dynamic electric power management system based on GIS[J]. Application of Electronic Technique, 2008, 34(3):105-107.

[4]Juan R. Personalized Service Model System Design of College Education Management System Based on Web Data Mining[J]. Computer Study, 2010.

[5]Wei F, Hong L I. The Analysis and Design of Management System of Supermarket Dispatching Based on UML[J]. Computer Knowledge \& Technology, 2009.

[6]Wen Y, Cui J, Zhang J, et al. Design of a Security Risk Management System for Power System Dispatching and Operation Part One Concepts and Design of Architecture and Function[J]. Automation of Electric Power Systems, 2013. 